\title{
pneumonia
}

Review

\section{The diagnosis of pneumonia requires a chest radiograph (x-ray) - yes, no or sometimes?}

\section{Dan Wootton ${ }^{\mathrm{a}, \mathrm{b}}$, Charles Feldman ${ }^{\mathrm{c}}$}

anstitute of Infection and Global Health, University of Liverpool, United Kingdom; ${ }^{b}$ Department of Respiratory Research, Aintree University Hospitals NHS Foundation Trust, United Kingdom; 'Division of Pulmonology, Department of Internal Medicine, Charlotte Maxeke Academic Hospital, Faculty of Health Sciences, University of the Witwatersrand, Johannesburg, South Africa

Corresponding author: Charles Feldman, Division of Pulmonology, Department of Internal Medicine, Charlotte Maxeke Johannesburg Academic, Hospital, Faculty of Health Sciences, University of the Witwatersrand, Johannesburg, South Africa. Phone: 011488 3840. Email: Charles.feldman@wits.ac.za

Author contribution: All authors met authorship criteria. All authors contributed equally to the writing of the first draft of the manuscript and writing of the manuscript. All authors critically reviewed the manuscript for important intellectual content. All authors agreed with the manuscript results and conclusions.

Received Apr 4, 2014; Accepted May 15, 2014; Published Jun 19, 2014

Citation: Wootton DG, Feldman C. The diagnosis of pneumonia requires a chest radiograph (x-ray) - yes, no or sometimes? pneumonia 2014;5:1-7

\begin{abstract}
Community-acquired pneumonia (CAP) remains a common condition associated with considerable morbidity and mortality. Outcome is improved by early recognition and rapid institution of empirical antibiotic therapy. A number of international guidelines recommend a chest radiograph ( $x$-ray) is obtained when pneumonia is suspected; the argument forwarded is that chest radiographs are relatively inexpensive and enable pneumonia (lung consolidation) to be confirmed or excluded. But, radiographs are not available in the community setting and introduce a delay in diagnosis and treatment. For these reasons, in mild CAP treated by primary care, guidelines suggest criteria for clinical diagnosis. However, there is debate as to whether clinical features alone are sufficiently reliable to support a diagnosis of CAP with some suggesting diagnostic precision is improved by chest radiographs. Conversely, several studies have demonstrated a lack of agreement in the interpretation of chest radiographs bringing their role as the ultimate arbiter of diagnosis into question. Below we debate the diagnostic role of the humble chest radiograph in the context of suspected CAP.
\end{abstract}

Keywords: pneumonia, chest x-ray, chest radiograph, diagnostic criteria, clinical diagnosis 


\section{Introductory statement}

\subsection{The case for (Feldman)}

CAP continues to be associated with a considerable burden of disease throughout the world [1-4]. Given the substantial ongoing morbidity and mortality it causes, it is not surprising that a number of guidelines have been developed in the different regions of the world describing what is considered the optimal management of this infection in the hope of improving the patient outcome [5-7]. Clearly one of the initial considerations in managing a patient with suspected CAP is to confirm that diagnosis, and to this end, the chest radiograph is universally considered to be the "gold standard" (1, 8-10]. The argument forwarded is that chest radiology is a relatively inexpensive procedure and is the only way in which pneumonia can be definitely confirmed or excluded.

Yet there are discrepancies in the different guideline recommendations with regard to the need for a routine chest radiograph. The current guideline from North America and the earlier European guideline indicated that if pneumonia was suspected a routine chest radiograph should be performed to confirm the diagnosis $[5,6]$. However, the British Thoracic Society guideline indicates that while all patients admitted to hospital with suspected CAP need a chest radiograph, in the community it is unnecessary for a chest radiograph to be done unless the diagnosis is in doubt, or clinical progress is not being made on treatment of a patient with suspected CAP, or if the patient is likely to have an underlying pathology, such as lung cancer [7]. Similarly, the more recent European guideline does not recommend a routine chest radiograph in the community setting but indicates that it could be done in cases in whom, despite c-reactive protein (CRP) testing, there is persistent doubt as to whether the diagnosis is pneumonia or another respiratory tract infection, and also in patients with suspected aspiration pneumonia [11].

In all guidelines there is a recommendation for routine chest radiology for all pneumonia patients admitted to hospital, and this appears to be relatively straightforward. However, it has been suggested by some that in the community, milder cases of infection treated as outpatients could be diagnosed on clinical grounds alone without the performance of chest imaging [12]. Reasons given for this approach include the cost of radiology, the inconvenience for the patient, the potential for delay in diagnosis while awaiting the chest radiograph and the lack of ready availability of routine radiology [12]. However, there is considerable debate about the accuracy of symptoms and signs alone in the diagnosis of CAP, with many suggesting that clinical features alone are not accurate enough and that a chest radiograph is an absolute requirement without which pneumonia cannot be adequately diagnosed or excluded.

\subsection{The case against (Wootton)}

To evaluate a diagnostic test such as a chest radiograph, we compare its performance to a "gold standard" [13]. If the gold standard definition of pneumonia includes a chest radiograph, then there are no comparisons to be made and there is no debate. Therefore, this discussion really asks, "What is pneumonia, and how accurately can a chest radiograph detect it?"

Our understanding of the term 'pneumonia' as an acute lower respiratory tract syndrome with systemic features has been consistent over thousands of years. In a translation of the Hippocratic Corpus written in the $5^{\text {th }}$ century BC, 'pneumonia' is referred to as "a disease named by the ancients":

\footnotetext{
... if fever be acute, and if there be pains on either side, or in both, and...if cough be present, and the sputa expectorated be of a blond or livid colour...[14].
}

This is remarkably similar to the present day British Thoracic Society's case definition.:

Symptoms of an acute lower respiratory tract illness...new focal chest signs on examination. At least one systemic feature...[7].

Most of our understanding of the pathophysiology of pneumonia derives from animal studies [15]. In those studies respiratory tract infection is induced experimentally and the development of pneumonia is suspected by observing a range of 'clinical' features such as fever, reduced activity, weight loss and laboured breathing and then confirmed post-mortem by examination of the lungs [16]. The post mortem pathology that underlies this syndrome has been defined as an acute inflammatory alveolar infiltrate caused by infection of the lung [15]. Inflammatory alveolar infiltrates driven by processes other than infection also occur and these borrow the term 'pneumonia'. Thus confirmation of the presence of a microbe and proof of a causal relationship with the inflammatory process would also be necessary to complete a diagnosis. Combining these elements, pneumonia is: a), lung infection, leading to b), lung inflammation, leading to c), respiratory and systemic symptoms.

Currently there is no single test that can reliably rule in or out all these features of pneumonia. In practice clinicians rely on clinical judgement supported, where available, by a range of 'tests' in order to come to a working diagnosis that enables us to arrive at a management plan. So to what extent can a chest radiograph help? Of the three diagnostic elements listed above it is only fair to ask the radiograph to contribute to b) and answer the question, "Is there lung inflammation?" Obviously this is an unfair challenge; a range of underlying mechanisms, some inflammatory, 
some not, can give rise to the same radiological appearances [17]. Moreover, even in a previously healthy individual, the radiograph has technically limited resolving power to detect inflammation at the alveolar level. More importantly the interpretation of the image is often difficult and frequently inconsistent. In this context, the chest radiograph demonstrates none of the performance characteristics that would be associated with a diagnostic test and therefore whilst it is an important tool to help manage some patients with possible respiratory infection, it should not be required for the 'diagnosis' of pneumonia which is a clinical syndrome.

\section{Criteria used by clinicians to diagnose pneumonia}

\subsection{The need for a chest radiograph (Feldman)}

Clearly an important issue in outpatient settings is the challenge that it poses on clinicians as to how to diagnose pneumonia without the ready availability of chest radiology. One study investigated the criteria used by primary care physicians to diagnose pneumonia, as opposed to acute bronchitis or upper respiratory tract infections, in the outpatient setting [18]. For the diagnosis of pneumonia the presence of crackles, temperature $\geq 37.8{ }^{\circ} \mathrm{C}$, chest pain, dyspnoea, rhonchi, rapid heart rate and respiratory rate and rhinorrhoea (absence of the latter) best explained the variation in pneumonia diagnosis; the presence of crackles, chest pain and temperature $\geq 37.8{ }^{\circ} \mathrm{C}$ best predicted the ordering of a chest radiograph (although $35 \%$ of cases diagnosed as pneumonia had a negative chest radiograph), and abnormal breath sounds were the best predictor for the prescribing of an antibiotic [18]. From the study it became clear that these primary care clinicians were not using established clinical prediction rules (see below) consistently in their practice, and furthermore, even among those patients diagnosed as having acute bronchitis alone, 93\% were prescribed antibiotics. It is also important to recognise that there is considerable interobserver variation in the recording of symptoms and also a high degree of interobserver error in the physical examination of the chest [1]. For many of these reasons it is the author's belief that a chest radiograph should be performed in patients for suspected CAP, if at all possible.

\subsection{The rebuttal (Wootton)}

To summarise the argument above, since clinical assessment is prone to variation, the radiograph should decide the diagnosis, presumably because it is believed to be more consistent. If we take this to its logical conclusion, why not do away with clinical assessment altogether? Perhaps patients with a cough should present to radiology clinics, then take the yes/no result to a pharmacy to be issued with either amoxicillin or linctus. This scenario has been tested by a number of studies that have examined the consistency of chest radiograph interpretation in the context of possible pneumonia. One way to evaluate a diagnostic test is to measure the level of agreement with respect to the interpretation of the result by calculating the kappa statistic. A kappa score less than 0.2 represents poor agreement that is little better than chance with 0.2-0.39 being regarded as fair, $0.4-0.59$ as moderate, $0.6-0.79$ as good and $>0.8$ as excellent [19]. In a study measuring the levels of agreement in radiograph interpretation between emergency physicians and radiologists, agreement with respect to pneumonia produced kappa $=0.395$ [20]. In another study, agreement between radiologists was kappa $=0.52$, training grade doctors kappa $=0.47$ and consultant physicians kappa $=0.34$ [21]. A study based in primary care in the Netherlands revealed kappa $=0.53$ for radiologists [22]. The largest published study of inter-observer agreement in the interpretation of chest radiograph in the context of possible pneumonia revealed radiologists had kappa $=0.38$ [23]. All studies revealed that disagreements, even among senior radiologists, were common, and in some studies, disagreements were more frequent than agreements; outside of the context of a study the situation is unlikely to be better. Clearly much of this inconsistency will be centred on certain chest radiographs which are particularly difficult to interpret, but in those cases, if the decision to diagnose pneumonia was based on the chest radiograph alone, we could save some time and money by flipping a coin.

\section{How accurate is clinical diagnosis?}

\subsection{Clinical examination (Feldman)}

Despite some studies suggesting that general practitioners may be accurate in diagnosing which patients with cough may have pneumonia and which patients require a chest radiograph [24], most investigations suggest that clinical judgement alone is relatively inaccurate and that history and clinical examination alone do not provide a high level of certainty with regard to the diagnosis of CAP $[1,8,25]$. For example, one investigation suggested that while primary care/emergency room physicians may have a reasonable ability to rule out radiologically-confirmed pneumonia on clinical grounds, in many cases in whom they consider to have pneumonia, this diagnosis is not confirmed on chest radiograph (sensitivity $74 \%$ [49-90\%], specificity $84 \%$ [78$88 \%$ ], negative predictive value 97\% [94-99\%], positive predictive value $27 \%$ [16-42\%]) [26].

In another study, 52 patients presenting with symptoms of an acute respiratory tract infection were recruited and a detailed pulmonary examination was undertaken by at least two of the three board-certified physicians, blinded to the history, vital signs or chest radiographic findings [25]. The chest radiographs were interpreted by a board certified radiologist and for the purposes of the study, pneumonia was defined as the presence of a radiographic infiltrate in the lung parenchyma, which was 
considered to be the "gold standard". Of the patients, 24 were radiologically diagnosed as having pneumonia. The 28 patients without pneumonia were considered to have either acute bronchitis or an exacerbation of chronic bronchitis. The two most common abnormal clinical findings found were crackles (22-65\%) and bronchial breath sounds (8-43\%). The sensitivity and specificity for clinical diagnosis of pneumonia varied between $0.47-0.69$ and $0.58-0.75$, respectively, for the three physicians. There were interobserver variations in the interpretation. The authors concluded that physical examination had a modest ability to predict the presence of pneumonia which alone was not sufficient to confirm or exclude the presence of this infection, and that when this diagnosis is suspected a chest radiograph remains the best test [25].

A further study in general practice investigated the value of typical symptoms and abnormal chest signs for the diagnosis of pneumonia in 402 adult patients presenting with an acute respiratory tract infection in whom the clinical features were compared with chest radiographs [27]. A radiological diagnosis of pneumonia was confirmed in 20 patients. The general practitioners in the study diagnosed pneumonia in only seven of these 20 patients and additionally in 22 patients who had normal chest radiographs. The likelihood ratio (LR) of pneumonia increased with increasing intensity of the symptoms. The LR of crackles was 3.7, which when evaluated against the doctors' diagnosis as a reference standard reached a LR of 14.8. The typical symptoms reached much lower LRs when evaluated against the radiographic reference standard. The authors concluded that crackles and other abnormal chest findings are interpreted too frequently as features of pneumonia, while the importance of typical symptoms was often underestimated [27].

\subsection{Clinical prediction rules (Feldman)}

In order to help support the clinical diagnosis of pneumonia, a myriad of clinical prediction rules have been developed [28-31]. In one study whose purpose it was to develop a prediction rule for the use of the chest radiograph in patients with acute respiratory symptoms, it was noted that positive vital sign and physical examination findings were useful as a screening tool for CAP, with a sensitivity of $95 \%$ and a specificity of $56 \%$ [29]. These investigators suggested, therefore, that in symptomatic patients presenting with acute respiratory symptoms, but having normal vital signs and physical examination, a chest radiograph may be unnecessary. However, because in $5 \%$ of those cases the diagnosis of CAP would be missed, this approach could only be used in patients with reliable follow-up and low likelihood of morbidity if CAP was not detected. Graffelman and colleagues [31], however, based on their assessment of the performance of these rules, suggested that models established on these clinical features do not reliably predict the presence of pneumonia. Clearly one issue that needs highlighting is that even among physicians there is poor agreement about the presence or absence of particular lung sounds and only $55 \%$ of the time was there complete agreement about the clinical findings [32].

One study evaluated the predictive value of several clinical variables for the diagnosis of pneumonia in adults presenting with acute respiratory symptoms [33]. Of 464 patients who had had a chest radiograph, pneumonia was confirmed in 129 (27.8\%). None of the symptoms, signs or laboratory findings studies reliably predicted pneumonia; however, absence of auscultatory findings on chest examination excluded pneumonia with a $95 \%$ certainly. In one investigation, 26 (22\%) of patients with pneumonia had a completely normal chest examination, whereas abnormal vital signs (temperature $>37.0^{\circ} \mathrm{C}$; pulse rate $>100$ beats/min, or respiratory rate $>20$ breaths/min) were $97 \%$ sensitive for the diagnosis of pneumonia [34]. In an even more recent study of European patients presenting with acute cough, general practitioners recorded whether pneumonia was a consideration or not following clinical evaluation [35]. Of the 2,810 patients, 140 (5\%) had radiographic evidence of pneumonia, of whom only 41 (29\%) had been clinically diagnosed, while 31 (1\%) had received a clinical diagnosis of pneumonia that was not confirmed on radiology, and overall, only $57 \%$ of clinically suspected pneumonias were confirmed radiographically. In cases with radiologically proven pneumonia who were not suspected on clinical grounds, less severe symptoms had been present $(p<0.05)$ [35].

\subsection{The rebuttal (Wootton)}

The studies cited in the above two paragraphs compare agreement between a clinical diagnosis and chest radiograph findings and conclude that there is very little concord. This is unsurprising given what we have learnt about the level of agreement regarding the interpretation of chest radiographs and the subjectivity of clinical assessment. These findings are consistent with practical clinical experience. It is not uncommon to assess a patient with clinical signs of pneumonia in whom the radiograph is initially normal but evolves to become abnormal a day or so later; or the patient with shortness of breath and unilateral pleuritic chest pain who has a normal chest radiograph and goes on to have a CT pulmonary angiogram that reveals an area of consolidation behind the heart, in the radiographs blind spot. Practically, we have to accept the diagnostic limitations of imaging and focus on the key management decisions. This author is of the belief that clinical judgement, despite its imperfections, has a key role to play in the context of patients with respiratory tract infection. Next time I am hypoxic - with cough, unilateral pleuritic chest pain, pyrexia, tachycardia and my doctor hears crackles in my chest - I will be perfectly happy to take the antibiotics my general practitioner prescribes me. And when I am admitted to hospital and my chest radiograph is reported as clear, I would be fascinated to know the 
diagnosis; acute bronchitis....really? The problem here is one of nomenclature. Even if the practical problems of making chest radiographs more widely available could be overcome, if we insist on chest radiograph findings to define pneumonia then we condemn some patients to a diagnostic no-man's land between acute bronchitis and pneumonia. In the acute situation this ambiguity can lead to delays in initiating therapy with adverse consequences.

\subsection{Value of the chest radiograph (Feldman)}

Not surprisingly many consider that chest radiology, which is a relatively inexpensive test, plays a fundamental and important role in the diagnosis of pneumonia, together with clinical assessment and sometimes appropriate microbiological testing [36]. Its primary purpose is to diagnose or exclude pneumonia, but it will also show the extent of the pneumonia, the presence or absence of associated comorbid conditions or complications, all of which may act as prognostic indicators, and it can also be used for subsequent follow-up to check for resolution [37]. The chest radiograph is much less helpful in suggesting the likely microbial aetiology of CAP although sometimes specific patterns are seen and may be helpful particularly in immunocompromised individuals [36-38]. Certainly, in the case of patients admitted to hospital there is evidence that the early performance of a chest radiograph is associated with clinical benefit, including a significantly shorter hospital length of stay and antibiotic use after radiology [39].

While some guidelines do advocate routine chest radiology for all cases with suspected CAP, even those guidelines that do not recommend routine radiology propose definitive criteria and/or situations in which a chest radiograph should be done [10, 29, 34]. For example, the American College of Radiology recommends that chest radiographs should be performed in patients with an acute respiratory illness and any of the following: age $>40$ years, dementia, positive physical examination, haemoptysis, leucocytosis, hypoxaemia, or other risk factors such as coronary artery disease, congestive heart failure and drug-induced respiratory failure [10]. Others have recommended a chest radiograph in all patients with an acute respiratory illness and the presence of at least one abnormal vital sign (temperature $>37.8^{\circ} \mathrm{C}$; pulse rate $>100$ beats/min, or respiratory rate $>20$ breaths/min) [34].

O'Brien's study [29] suggested that chest radiographs were unnecessary in patients with acute respiratory symptoms and normal vital signs and physical examination (sensitivity 95\%). However, since this approach would miss $5 \%$ of pneumonias, they recommend that this should be reserved only for cases with reliable follow up and also a low likelihood of morbidity if pneumonia was missed [29]. Furthermore, Ebell [40] suggested a simple rule for determining the need for chest radiography in a patient with acute respiratory illness that others have adopted [41], which recommend that chest radiology be performed in any of the following situations:

- In any patient demonstrating at least one of the following abnormal vital signs:

- Temperature $>37.8^{\circ} \mathrm{C}$

- Heart rate $>100$ beats/minute

- Respiratory rate $>20$ breaths/minute

- In any patient with at least two of the following clinical findings:

- Decreased breath sounds

- Crackles

- Absence of asthma

\subsection{The rebuttal (Wootton)}

A chest radiograph may be a valuable tool in the ongoing management of some patients with lower respiratory tract infection. In some patients a chest radiograph may be useful in screening for pre-disposing pathology such as lung cancer and identifying complications of the infection, such as empyema, but both those conditions require further imaging or tests for diagnosis. However, in the context of lower respiratory tract infection there is little evidence that performing chest radiographs alters meaningful outcomes for ambulatory patients [42]. For example, in a prospective study of lower respiratory tract infection in the community, "half of the patients with changes on the chest radiograph recovered without antibiotics" [43]. This suggests that even if an early chest radiograph was available for all patients in primary care it would be very unlikely to affect outcome. In the study by Bewick et al. [39] based in a United Kingdom hospital practice, a reduction in time to first antibiotic (TFA) (TFA $<4$ hours versus TFA $>4$ hours) was associated with reduced length of stay ( 5.63 days versus 8.07 days). That result suggests that performing a chest radiograph early leads to reduced length of stay, not because radiographs cure pneumonia, but because the earlier the chest radiograph is performed the earlier the antibiotics can be delivered. What we don't know from that study is what happened to the patients who had lower respiratory tract infections but whose chest radiograph was reported as normal. How many of them got antibiotics and what were their length of stay or re-admission rate? In the era of enhanced antibiotic stewardship, it should be possible to commence antibiotics pending further tests (which might include a chest radiograph) and to review the requirement for ongoing antibiotic treatment in the light of further information and clinical response.

\section{Conclusion}

\subsection{Feldman}

From the preceding discussion, it is apparent that the clinical features alone, including both the symptoms and 
the physical findings together, are not sufficient for the accurate diagnosis or exclusion of CAP. Part of the reason for these observations may be the inaccuracy of clinical examination with considerable interobserver variation in the recording of symptoms and also a high degree of interobserver error in the physical examination of the chest $[1,25,32]$. Furthermore, none of the clinical findings have been found to have $100 \%$ sensitivity and $100 \%$ specificity in ruling out pneumonia in any of the studies. Therefore, there will be those patients in whom the diagnosis of CAP will be missed, particularly among those with milder symptoms, who are more likely to be among those treated in the community $[28,35]$. Furthermore, there will also be those in whom CAP will be diagnosed clinically, in the absence of radiologically confirmed infection [26, 27], in whom antibiotics may be given, perhaps unnecessarily. For these reasons it is widely recommended that routine radiology be performed in any patient suspected on any clinical grounds of having CAP.

\subsection{Wootton}

Pneumonia is an old term. It describes a syndrome that falls within the spectrum of lower respiratory tract infections and is distinguished by the presence of systemic features. It is caused by infection and inflammation of the lung and this can be suspected from the history and sometimes detected by clinical examination and/or a chest radiograph. However, since a chest radiograph cannot reliably rule in or rule out lung infection it could not be regarded as a diagnostic test. The clinical priority is not whether the diagnostic label should be 'pneumonia' but whether the patient requires antibiotic therapy and where they can be best cared for. These two management decisions are based upon two further questions - "Is this a bacterial infection?" and "How sick is the patient?," and a radiograph can answer neither. Once these decisions have been made a chest radiograph may be useful in further management and follow-up, but is not required in all cases.

Disclaimer: The views expressed in this article do not necessarily represent the personal views of the authors, but have been written to stimulate debate.

Funding: CF is supported by the National Research Foundation, South Africa. DW was supported by a Doctoral Research Fellowship from the National Institute of Health Research (NIHR), United Kingdom, during the preparation of the manuscript. The funders had no role in study design, data collection and analysis, decision to publish, or preparation of the manuscript.

Competing interests: All authors declare no competing interests.

Provenance and peer review: Commissioned; no funding has been requested or received by the authors for the preparation of the manuscript; externally peer reviewed.

Copyright: This is an open-access article distributed under the terms of the Creative Commons Attribution License, which permits unrestricted use, distribution, and reproduction in any medium, provided the original author and source are credited.

\section{References}

1. Metlay JP, Kapoor WN,Fine MJ. Does this patient have community-acquired pneumonia?: Diagnosing pneumonia by history and physical examination. JAMA. 1997;278(17):1440-45.http://dx.doi.org/10.1001/ jama.278.17.1440

2. Welte T, Torres A, Nathwani D. Clinical and economic burden of community-acquired pneumonia among adults in Europe. Thorax. 2012;67(1):71-9. http://dx.doi. org/10.1136/thx.2009.129502

3. File T, Marrie T. Burden of community-acquired pneumonia in North American adults. Postgrad Med 2010;122(2):13041. http://dx.doi.org/10.3810/pgm.2010.03.2130

4. Irfan M, Farooqi J, Hasan R. Community-Acauired Pneumonia. Curr Opin Pulm Med 2013;3:198-208. http:// dx.doi.org/10.1097/MCP.0b013e32835f1d12

5. Woodhead M, Blasi F, Ewig S, Garau J, Huchon G, leven $M$, et al. Guidelines for the management of adult lower respiratory tract infections - Full version. Clin Microbiol Infect 2011;17:E1-E59. http://dx.doi.org/10.1111/j.14690691.2011.03602.x

6. Mandell LA, Wunderink RG, Anzueto A, Bartlett JG, Campbell GD, Dean NC, et al. Infectious Diseases Society of America/American Thoracic Society Consensus Guidelines on the Management of Community-Acquired Pneumonia in Adults. Clin Infect Dis 2007;44:S27-S72. http://dx.doi. org/10.1086/511159

7. Lim WS, Baudouin SV, George RC, Hill AT, Jamieson C, Le Jeune I, et al. BTS guidelines for the management of community acquired pneumonia in adults: update 2009. Thorax 2009;64 Suppl 3:iii1-iii55. http://dx.doi. org/10.1136/thx.2009.121434

8. Metlay JP, Fine MJ. Testing strategies in the initial management of patients with community-acquired pneumonia. Ann Intern Med 2003;138(2):109-18. http:// dx.doi.org/10.7326/0003-4819-138-2-200301210-00012

9. Armitage $K$, Woodhead $M$. New guidelines for the management of adult community-acquired pneumonia. Curr Opin Infect Dis 2007;20(2):170-6. http://dx.doi. org/10.1097/QCO.0b013e3280803d70

10. Washington L, Palacio D. Imaging of bacterial pulmonary infection in the immunocompetent patient. Semin Roentgenol 2007;42(2):122-45. http://dx.doi. org/10.1053/j.ro.2006.08.008

11. Woodhead M, Blasi F, Ewig S, Garau J, Huchon G, leven M, et al. Guidelines for the management of adult lower respiratory tract infections - Summary. Clin Microbiol Infect 2011;17:1. http://dx.doi.org/10.1111/j.1469-0691.2011.03602.x

12. Newbegin C, Macfarlane J. Diagnosing pneumonia accurately in the community--is it necessary? Prim Care Respir J 2010;19(3):200-01. http://dx.doi.org/10.4104/pcrj.2010.00051

13. Bossuyt PM, Reitsma JB, Bruns DE, Gatsonis CA, Glasziou PP, Irwig LM, et al. Towards complete and accurate reporting of studies of diagnostic accuracy: the STARD initiative. Vet Clin Pathol 2007;36(1):8. http://dx.doi.org/10.1111/j.1939165X.2007.tb00175.x

14. Murray J. The Captain Of The Men Of Death: History Of Pneumonia. In: Marrie TJ, editor. Community-Acquired Pneumonia New York: Springer; 2001. p. 1.

15. Wootton DG, Aston SJ, Gordon SB. The Pathophysiology Of Pneumococcal Pneumonia. In: Chalmers JD, Aliberti S, 
Pletz M, editors. European Respiratory Society Monograph: Community-Acquired Pneumonia. 63rd ed.: European Respiratory Society; 2014. p. 42-63. http://dx.doi. org/10.1183/1025448x.10003313

16. Medina E. Murine model of pneumococcal pneumonia. Mouse Models for Drug Discovery: Methods and Protocols 2010;602:405-10. http://dx.doi.org/10.1007/978-1-60761058-8 22

17. Kim TH, Kim SJ, Ryu YH, Chung SY, Seo JS, Kim YJ, et al. Differential CT features of infectious pneumonia versus bronchioloalveolar carcinoma (BAC) mimicking pneumonia. Eur Radiol 2006;16(8):1763-8. http://dx.doi.org/10.1007/ s00330-005-0101-5

18. Evertsen J, Baumgardner DJ, Regnery A, Banerjee I. Diagnosis and management of pneumonia and bronchitis in outpatient primary care practices. Prim Care Respir J 2010;19(3):23741. http://dx.doi.org/10.4104/pcrj.2010.00024

19. Chmura Kraemer H, Periyakoil VS, Noda A. Kappa coefficients in medical research. Stat Med 2002;21(14):2109. http:// dx.doi.org/10.1002/sim.1180

20. Al Aseri Z. Accuracy of chest radiograph interpretation by emergency physicians. Emergency Radiology 2009;16(2):11114. http://dx.doi.org/10.1007/s10140-008-0763-9

21. Singh B, Curtis J, Gordon SB, Diggle PJ, Wootton DG. Junior Doctors' Interpretation of CXRs is More Consistent than Consultants in the Context of Possible Pneumonia. Thorax 2011:A169-A170. http://dx.doi.org/10.1136/thoraxjnl-2011201054c.251

22. Hopstaken R. Inter-observer variation in the interpretation of chest radiographs for pneumonia in community-acquired lower respiratory tract infections. Clin Radiol 2004;59(8):743. http://dx.doi.org/10.1016/j.crad.2004.01.011

23. Albaum MN, Hill LC, Murphy M, Li Y, Fuhrman CR, Britton $\mathrm{CA}$, et al. Interobserver reliability of the chest radiograph in community-acquired pneumonia. Chest 1996;110(2):34350. http://dx.doi.org/10.1378/chest.110.2.343

24. Blaeuer SR, Bally K, Tschudi P, Martina B, Zeller A. Acute cough illness in general practice - predictive value of clinical judgement and accuracy of requesting chest $x$-rays. Praxis (Bern 1994) 2013;102(21):1287-92. http://dx.doi. org/10.1024/1661-8157/a001437

25. WipfJE, Lipsky BA, HirschmannJV, BoykoEJ, TakasugiJ, Peugeot $\mathrm{RL}$, et al. Diagnosing pneumonia by physical examination: relevant or relic? Arch Intern Med 1999;159(10):1082-7. http://dx.doi.org/10.1001/archinte.159.10.1082

26. Lieberman D, Shvartzman P, Korsonsky I, Lieberman D. Diagnosis of ambulatory community-acquired pneumonia. Comparison of clinical assessment versus chest $\mathrm{X}$-ray. Scand J Prim Health Care 2003;21(1):57-60. http://dx.doi. org/10.1080/02813430310000582

27. Melbye $H$, Straume B, Aaseb $\varnothing$ U, Dale K. Diagnosis of pneumonia in adults in general practice. Relative importance of typical symptoms and abnormal chest signs evaluated against a radiographic reference standard. Scand J Prim Health Care 1992;10(3):226-33. http://dx.doi. org/10.3109/02813439209014066

28. Pisarik P, Montoya C, Malloy ED, Pisarik P, Montoya C. Clinical inquiries. When should a chest $\mathrm{x}$-ray be used to evaluate acute-onset productive cough for adults? J Fam Pract 2005;54(12):1081-83.

29. O'Brien W, Rohweder D, Lattin G, Thorrnton J, Dutton $\mathrm{J}$, Ebert-Long $\mathrm{D}$, et al. Clinical Indicators of radiographic findings in patients with supected community acquired pneumonia: Who needs a chest x-ray? J Am Coll Radiol 2006;3:703-6. http://dx.doi.org/10.1016/j.jacr.2006.02.007

30. Cals J, Hopstaken R. Lower respiratory tract infections: Treating patients or diagnoses? J Fam Pract 2006;55(6):545-6.

31. Graffelman AW, le Cessie S, Neven AK, Willemssen FEJA, Zonderland HM, van dB. Can history and exam alone reliably predict pneumonia? J Fam Pract 2007;56(6):465-70.

32. Spiteri M, Cook D, Clarke S. Reliability of eliciting physical signs in examination of the chest. Lancet 1998;331(8590):873-5. http://dx.doi.org/10.1016/S01406736(88)91613-3

33. Heckerling P. The need for chest roentgenograms in adults with acute respiratory illness. Arch Intern Med 1986;146:1321-4. http://dx.doi.org/10.1001/archinte.1986.00360190085012

34. Gennis P, Gallagher J, Falvo C, Baker S, Than W. Clinical criteria for the detection of pneumonia in adults: guidelines for ordering chest roentgenograms in the emergency department. J Emerg Med 1989;7(3):263-8. http://dx.doi. org/10.1016/0736-4679(89)90358-2

35. van Vugt S,F., Verheij TJM, de Jong P,A., Butler CC, Hood $\mathrm{K}$, Coenen $\mathrm{S}$, et al. Diagnosing pneumonia in patients with acute cough: clinical judgment compared to chest radiography. Eur Respir J 2013;42(4):1076-82. http://dx.doi. org/10.1183/09031936.00111012

36. Sharma S, Maycher B, Eschun G. Radiological imaging in pneumonia: recent innovations. Curr Opin Pulm Med 2007;13(3):159-69. http://dx.doi.org/10.1097/ MCP.0b013e3280f3bff4

37. Reynolds JH, Banerjee AK. Imaging pneumonia in immunocompetent and immunocompromised individuals. Curr Opin Pulm Med 2012;18(3):194-201. http://dx.doi. org/10.1097/MCP.0b013e328351f953

38. Boersma WG, Daniels JMA, Löwenberg A, Boeve W, van de Jagt $E$,J. Reliability of radiographic findings and the relation to etiologic agents in community-acquired pneumonia. Respir Med 2006;100(5):926-32. http://dx.doi. org/10.1016/j.rmed.2005.06.018

39. Bewick T, Greenwood S, Wei SL. The impact of an early chest radiograph on outcome in patients hospitalised with communityacquired pneumonia. Clinical Medicine 2010;10(6):563-7. http://dx.doi.org/10.7861/clinmedicine.10-6-563

40. Ebell $\mathrm{MH}$. Predicting pneumonia in adults with respiratory illness. Am Fam Physician 2007;76(4):560-2.

41. Watkins RR, Lemonovich TL. Diagnosis and management of community-acquired pneumonia in adults. Am Fam Physician 2011;83(11):1299-1306.

42. Cao AM, Choy JP, Mohanakrishnan LN, Bain RF, van Drie ML. Chest radiographs for acute lower respiratory tract infections. Cochrane Database Syst Rev 2013;12:CD009119.

43. Woodhead MA, Macfarlane JT, McCracken JS, Rose DH, Finch RG. Prospective study of the aetiology and outcome of pneumonia in the community. Lancet 1987;1(8534):671-4. http://dx.doi.org/10.1016/S0140-6736(87)90430-2 